\title{
MAXIMAL COMPACT NORMAL SUBGROUPS AND PRO-LIE GROUPS
}

\author{
R. W. BAGLEY AND T. S. WU
}

\begin{abstract}
We are concerned with conditions under which a locally compact group $G$ has a maximal compact normal subgroup $K$ and whether or not $G / K$ is a Lie group. If $G$ has small compact normal subgroups $K$ such that $G / K$ is a Lie group, then $G$ is pro-Lie. If in $G$ there is a collection of closed normal subgroups $\left\{H_{\alpha}\right\}$ such that $\bigcap H_{\alpha}=e$ and $G / H_{\alpha}$ is a Lie group for each $\alpha$, then $G$ is a residual Lie group. We determine conditions under which a residual Lie group is pro-Lie and give an example of a residual Lie group which is not embeddable in a pro-Lie group.
\end{abstract}

DEFINITION 1. A locally compact topological group $G$ is an $L$-group if, for each neighborhood $U$ of the identity and compact subset $C$ of $G$, there is a neighborhood $V$ such that $g H g^{-1} \cap C \subset U$ for every $g \in G$ and subgroup $H \subset V$.

DEFINITION 2. A topological group $G$ is pro-Lie if $G$ has small compact normal subgroups $K$ such that $G / K$ is a Lie group.

DEFINITION 3. A locally compact group $G$ is a residual Lie group if there is a collection of closed normal subgroups $\left\{H_{\alpha}\right\}$ such that $\bigcap H_{\alpha}=e$ and $G / H_{\alpha}$ is a Lie group for each $\alpha$.

It is easy to see that a residual Lie group is an $L$-group [1, Theorem 1.3 and its Corollary]. In this paper we obtain the following: If $G$ is an $L$-group and has an open normal subgroup $G_{1}$ such that $G_{1} / G_{0}$ is compact, then $G$ is pro-Lie. There exists a locally compact group $G$ with a maximal compact normal subgroup $K$ such that $G / K$ is not a Lie group. A residual Lie group is not necessarily embeddable by a continuous isomorphism in a pro-Lie group. A generalized FC-group has a maximal compact normal subgroup.

PROPOSITION 1. For a locally compact group $G$, the following are equivalent:

(1) $G$ has a compact normal subgroup $K$ such that $G / K$ is a Lie group.

(2) $G$ has a compact normal subgroup $K$ such that $G / K$ is locally connected.

(3) $G$ has an open normal subgroup $G_{1}$ such that $G_{1} / G_{0}$ is compact.

We denote the connected component of the identity of $G$ by $G_{0}$.

Proof. Obviously (1) implies (2). We assume that $K$ is compact normal and $G / K$ is locally connected. Thus $(G / K)_{0}=G_{0} / K$ is open in $G / K$. It follows that $K G_{0}=G_{1}$ is an open normal subgroup of $G$ and $G_{1} / G_{0}$ is compact. We complete the proof by showing that (3) implies (1). Assuming (3) we have a maximal compact normal subgroup $K$ of $G_{1}$ and $G_{1} / K$ is a Lie group [1, Proposition 2.6]. Since $K$ is maximal in $G_{1}$ and $G_{1}$ is normal in $G, K$ is normal in $G$. Since $G / G_{1}$ is discrete and $G_{1} / K$ is a Lie group, $G / K$ is a Lie group. 
THEOREM 2. For a locally compact group $G$, the following are equivalent:

(1) $G$ is pro-Lie.

(2) $G$ is an L-group and $G / G_{0}$ is pro-Lie.

(3) $G$ is an L-group and has a compact normal subgroup $K$ such that $G / K$ is locally connected.

(4) $G$ is an $L$-group and has an open normal subgroup $G_{1}$ such that $G_{1} / G_{0}$ is compact.

ProOF. Obviously (1) implies (3). The reverse implication follows from Proposition 1 and [1, Theorem 1.2 and Corollary 2]. Also by Proposition 1, (3) and (4) are equivalent. Since (1) obviously implies (2) we can complete the proof by showing that (2) implies (4). This follows immediately from $[\mathbf{1}$, Proposition 1.7 and Corollary 2].

If $G$ is a locally compact group and $K$ is a maximal compact normal subgroup, then there are some obvious cases when $G / K$ is a Lie group. For example:

(1) If there is an open normal subgroup $G_{1}$ such that $G_{1} / G_{0}$ is compact, then $G / K$ is a Lie group. Now $G_{1}$ has a maximal compact normal subgroup $K_{1}$ such that $G_{1} / K_{1}$ is a Lie group. Also $K_{1}$ is normal in $G$; thus, $G / K_{1}$ is a Lie group. It follows that, if $K$ is maximal compact normal in $G$, then $G / K$ is a Lie group.

(2) If $G / H$ is locally connected for some compact normal subgroup $H$, then $G / K$ is a Lie group.

(3) If $G / K$ is pro-Lie, then it is a Lie group.

By the semidirect product $H \times_{\eta} K$ of two groups $H$ and $K$ we mean the group determined by a homomorphism $\eta: K \rightarrow A(H)$, the automorphism group of $H$, with the group operation in $H \times_{\eta} K$ defined by $(h, k)\left(h_{1}, k_{1}\right)=\left(h \eta(k)\left(h_{1}\right), k k_{1}\right)$.

EXAMPLE 1. Let $G=\left(\sum_{i=-1}^{-\infty} K_{i}\right) \times\left(\prod_{i=1}^{\infty} K_{i}\right) \times_{\eta} Z$, where $K_{i}=\{0,1\}$ and the integer $n \in Z$ shifts coordinates $n$ places to the left. We assume $\sum K_{i}$ has the discrete topology and $\prod K_{i}$ has the product topology. It is easy to see that any nontrivial invariant subset $C$ of $G$ contains elements $\left(k(n), z^{j}\right)$, where the $n$th coordinate of $k(n)$ is 1 for infinitely many integers $n$, and $j$ is some fixed integer. Thus $C$ is not compact. It follows that the maximal compact normal subgroup of $G$ is the identity. Thus we have a compactly generated group with maximal compact normal subgroup $K$ such that $G / K$ is not a Lie group.

There are many examples of topological groups without maximal compact normal subgroups; for example, any discrete group with no maximal finite subgroup.

EXAMPLE 2. We give another example of a locally compact group $G$ which is not a Lie group and in which the identity is the maximal compact normal subgroup. Let $G=\sum_{i=1}^{\infty} H_{i} \times_{\eta} \prod_{i=0}^{\infty} K_{i}$, where $H_{i}=Z$, the additive group of integers, and $K_{i}=Z_{2}$, the multiplicative two-element group. The automorphism $\eta(k)$ of $\sum H_{i}$ is defined as $\eta(k)(h)_{i}=k_{i} h_{i}$. It is easy to see that if $\sum H_{i}$ has the discrete topology and $\prod K_{i}$ the product topology, $G$ has the desired properties.

DEFINITION. A locally compact group $G$ is a generalized $F C$-group if $G=A_{1} \supset$ $A_{2} \supset \cdots \supset A_{n} \supset A_{n+1}=e$, where each $A_{i}$ is a closed normal subgroup of $G$ and $A_{i} / A_{i+1}$ is a compactly generated FC-group.

We note that by [2, Corollary 3.9], every compactly generated FC-group has a compact normal subgroup such that the corresponding factor group is of the form $V \times Z^{k}$. 
THEOREM 3. If $G$ is a locally compact generalized $F C$-group, then $G$ has a maximal compact normal subgroup.

ProOF. We proceed by induction, noting that, for $n=1$, the conclusion follows from the corollary referred to above. Let $G=A_{1} \supset A_{2} \supset \cdots \supset A_{n+1}=e$, where each $A_{i} / A_{i+1}$ is a compactly generated FC-group. We assume that every generalized FC-group with sequence $\left\{A_{i}\right\}$ of shorter length has a maximal compact normal subgroup. Thus $G / A_{n}$ has a maximal compact normal subgroup $M$ and $A_{n}$ has a maximal compact normal subgroup $K$. Let $N=\pi^{-1}(M)$, where $\pi$ is the canonical mapping of $G$ onto $G / A_{n}$. Again, using [2, Corollary 3.9], we let $Q=Z\left(A_{n} / K, N / K\right)$ and note that $Q$ is a compactly generated central group. Thus $Q$ has a maximal compact normal subgroup. We let $P$ be the inverse image of this subgroup under the canonical mapping of $N$ onto $N / K$. We show that $P$ is the maximal compact normal subgroup of $G$. Let $L$ be any compact normal subgroup of $G$. Then $\pi(L) \subset M$ and $L \subset N$. Also $L / K \cap A_{n} / K=\{e\}$ since $A_{n} / K \cong V \times Z^{k}$. If follows that $L / K \subset Q$ and $L \subset P$ as desired.

DEFINITION 4. A topological group $G$ is an $N$-group if for every neighborhood $U$ of the identity and compact set $C$ of $G$, there is a neighborhood $V$ of the identity such that $g V g^{-1} \subset U \cup(G-C)$ for all $g \in G$. A group $G$ is a $S I N$-group if $G$ has small neighborhoods of the identity which are invariant under the inner automorphisms of $G$.

It is easy to see that, if a topological group $G$ can be embedded in an SIN-group, then $G$ is an $N$-group.

EXAMPLE 3. We construct an example of an $N$-group which is residual Lie and which cannot be embedded in a pro-Lie group. We begin with the group $F$ of $2 \times 2$ matrices of determinant equal to 1 . Let $H$ be the subgroup of $F$ of matrices of the form $\left(\begin{array}{ll}1 & c \\ 0 & 1\end{array}\right)$. If $N_{0}$ is a normal subgroup of $F \times F$ which contains all elements of the form $\left[A, A^{-1}\right]$, then $N_{0}$ contains all pairs of the form $[I, C]$, where $I$ is the identity and $C$ is an element of $H$. To show this, let $A=\left(\begin{array}{cc}a / y & 1 \\ 0 & y / a\end{array}\right)$ and $B=\left(\begin{array}{cc}1 / a & 0 \\ 0 & a\end{array}\right)$. Then

$$
\left[A B, A^{-1} B^{-1}\right]=\left[\left(\begin{array}{cc}
1 / y & a \\
0 & y
\end{array}\right),\left(\begin{array}{cc}
y & -1 / a \\
0 & 1 / y
\end{array}\right)\right]
$$

is in $N_{0}$. Thus, if

$$
A=\left(\begin{array}{cc}
a^{2}-1 / c a & a \\
0 & c a / a^{2}-1
\end{array}\right) \quad \text { and } B=\left(\begin{array}{cc}
c a / a^{2}-1 & -1 / a \\
0 & a^{2}-1 / c a
\end{array}\right)
$$

then $[A, B]$ is in $N_{0}$. It follows that $\left[I,\left(\begin{array}{ll}1 & c \\ 0 & 1\end{array}\right)\right]$ is in $N_{0}$ as desired, since $\left[I,\left(\begin{array}{ll}1 & c \\ 0 & 1\end{array}\right)\right]=$ $[A, B]\left[A^{-1}, A\right]$.

If $c>0$ and $C=\left(\begin{array}{ll}1 & c \\ 0 & 1\end{array}\right)$, then $[I, C]$ is the conjugate of $\left[I,\left(\begin{array}{ll}1 & 1 \\ 0 & 1\end{array}\right)\right]$ by $\left[A^{-1}, A\right]$, where

$$
A=\left(\begin{array}{cc}
\sqrt{c} & 1 \\
0 & 1 / \sqrt{c}
\end{array}\right) \text {. }
$$

If $c<0$, then $[I, C]$ is the conjugate of $\left[I,\left(\begin{array}{cc}1 & -1 \\ 0 & 1\end{array}\right)\right]$ by $\left[A^{-1}, A\right]$, where

$$
A=\left(\begin{array}{cc}
\sqrt{-c} & -1 \\
0 & 1 / \sqrt{-c}
\end{array}\right) \text {. }
$$

Thus, $N_{1}=I \times H$ has only three conjugacy classes relative to $N_{0}$. We show that $N_{0}$ cannot be embedded (isomorphically) in a compact group. 
LEMMA. If $G$ is a discrete group and $H$ is an infinite subgroup which has only finitely many conjugacy classes relative to $G$, then $G$ cannot be embedded in a compact group.

Proof. Suppose there exists an isomorphism $Q: G \rightarrow K$, a compact group. If $H_{0}$ is a conjugacy class of $H$ such that $H_{0} \neq\{e\}$, then there is a neighborhood $V$ of the identity of $K$ such that $Q\left(H_{0}\right) \cap V=\varnothing$. Because, if $Q\left(H_{0}\right)$ intersects every neighborhood of the identity of $K$, then for any neighborhood $W$ there is a neighborhood $V$ of the identity of $K$ such that $g V g^{-1} \subset W$. If $Q\left(H_{0}\right) \cap V \neq \varnothing$, then $Q\left(H_{0}\right) \subset W$. Consequently $Q\left(H_{0}\right)=e$ which is a contradiction. Since $H$ has only finitely many conjugacy classes, there is a neighborhood $U$ of the identity of $K$ such that $Q(H) \cap U=e$. Thus $Q(H)$ is discrete which contradicts the fact that $K$ is compact.

We are ready to construct the example referred to above. Let $F_{i}=F \times F$, where $F$ is the group of $2 \times 2$ matrices referred to above and $K_{i}=Z_{2}$ for each positive integer $i$. Let $G=\sum F_{i} \times_{\eta} \prod K_{i}$, where $\sum F_{i}$ is the direct sum with discrete topology, $\Pi K_{i}$ is the direct product with product topology, and $\eta$ is defined as follows: If the $i$ th coordinate of $k \in \prod K_{i}$ is 1 , then the $i$ th coordinate of $\eta(k)(f)$ is $\left(f_{2}, f_{1}\right)$, where the $i$ th coordinate of $f$ is $\left(f_{1}, f_{2}\right)$. If the $i$ th coordinate of $k$ is 0 , then, of course, $\eta(k)$ does not alter the $i$ th coordinate of $f \in \sum F_{i}$.

We note that any open normal subgroup of $G$ contains an isomorphic image of a subgroup $N_{0}$ of $F \times F$ such that $\left[A, A^{-1}\right] \in N_{0}$ for each $A \in F$. To see this we note that, if $k \in \prod K_{i}$ has $i$ th coordinate 1 and all other coordinates 0 , and $f \in \sum F_{i}$ has $i$ th coordinate $[A, I]$, and all other coordinates $[I, I]$, then $(f, 0) \in \sum F_{i} \times_{\eta} \prod K_{i}$ and $(f, 0)(I, k)(f, 0)^{-1}=\left(f^{\prime}, k\right)$, where $f^{\prime}$ has $i$ th coordinate $\left[A, A^{-1}\right]$ and all other coordinates $[I, I]$, where $I$ here is the identity of $F$.

To show that $G$ cannot be embedded in a pro-Lie group, assume there exists a continuous isomorphism $Q: F \rightarrow G_{1}$ and $G_{1}$ is pro-Lie. Let $K$ be a normal compact subgroup of $G_{1}$ such that $G_{1} / K$ is a Lie group. Then $G / Q^{-1}(K)$ is a Lie group, hence discrete since $G$ is totally disconnected. It follows that $Q^{-1}(K)$ is an open normal subgroup of $G$. This contradicts the Lemma. Thus, the existence of the continuous isomorphism $Q$ is contradicted.

Since a locally compact SIN-group is pro-Lie [2, Theorem 2.11], it follows from the above argument that $G$ cannot be embedded in a locally compact SIN-group.

In conclusion we see that $G$ is an $N$-group and residual Lie. Actually $G$ can be embedded in $\sum F_{i} \times_{\eta} \prod K_{i}$, where $\sum F_{i} \times \prod K_{i}$ has the relative product topology of $\prod F_{i} \times \prod K_{i}$ which is SIN but, of course, not locally compact.

\section{REFERENCES}

1. R. W. Bagley, T. S. Wu and J. S. Yang, Pro-Lie groups, Trans. Amer. Math. Soc. (to appear).

2. S. Grosser and M. Moskowitz, Compactness conditions in topological groups, J. Reine Angew. Math. 246 (1971), 1-40. 33124

Department of Mathematics, University of Miami, Coral Gables, Florida

Department of Mathematics and Statistics, Case Western Reserve UniverSity, ClEVELAND, OHIO 44106 